\title{
Erratum to: A novel secure and efficient hash function with extra padding against rainbow table attacks
}

\author{
Hyung-Jin Mun ${ }^{1} \cdot$ Sunghyuck Hong ${ }^{2} \cdot J^{\prime}$ ungpil Shin ${ }^{3}$
}

Published online: 1 July 2017

(C) Springer Science+Business Media, LLC 2017

\section{Erratum to: Cluster Comput}

\section{DOI: $10.1007 / \mathrm{s} 10586-017-0886-4$}

The original version of the article contained a mistake. Data in the Figure 6 has been missed. The correct Figure 6 has been updated with an erratum and original article is corrected.

\begin{tabular}{|c|c|c|}
\hline No & Id & Password \\
\hline 1 & 1q2w3e & 3fde6bb0541387e4ebdadf7c2ff31123 \\
\hline 2 & password1 & 7c6a180b36896a0a8c02787eeafb0e4c \\
\hline 3 & admin1234 & 751cb3f4aa17c36186f4856c8982bf27 \\
\hline 4 & $1234 \mathrm{abcd}$ & ef73781effc5774100f87fe2f437a435 \\
\hline 5 & asdfgh & a152e841783914146e4bcd4f39100686 \\
\hline
\end{tabular}

Fig. 6 Account information table seized by attacker

The online version of the original article can be found under doi:10.1007/s10586-017-0886-4.

\section{Sunghyuck Hong}

shong@bu.ac.kr

1 Department of Information \& Communication Engineering, Sungkyul University, Anyang 14097, Korea

2 Division of Information and Communication, Baekseok University, Cheonan 31065, Korea

3 School of Computer Science and Engineering, The University of Aizu, Aizu-Wakamatsu 965-8580, Japan 